\title{
The analysis of diesel engine intake system simulation based on multivariate joint optimization
}

\author{
REN Hongjuan, Wang Huilong, LUO Yiping \\ Shanghai University of Engineering and Science, Longteng Road 333, Songjiang District, Shanghai, China
}

Email address:

ren-hongjuan@163.com (Ren Hongjuan),m18818236190@163.com (Wang Huilong), lyp777@sina.com (Luo Yiping)

\section{To cite this article:}

Luo Yiping, Wang Huilong, Ren Hongjuan. The Analysis of Diesel Engine Intake System Simulation Based on Multivariate Joint Optimization. International Journal of Energy and Power Engineering. Vol. 3, No. 3, 2014, pp. 154-157.

doi: $10.11648 /$ j.ijepe.20140303.17

\begin{abstract}
The paper was based on multivariable joint optimization. It established an optimization process of diesel engine intake system. The simulation model was built in AVL-BOOST and tested by experiments. As the design variables of intake system parameters (diameter and length of intake duct and intake manifold) were used to orthogonal test. Through the calculation of the engine performance optimization, the sum of paper was the impact of key parameters of the intake system on the performance of engine, and the influence of variable parameters on the volumetric efficiency of engine. These works provided a theoretical reference of intake system optimization design.
\end{abstract}

Keyword: Diesel Engine, Intake System, AVL-BOOST, Volumetric Efficiency, Orthogonal Experiment Design, Multivariate Joint Optimization

\section{Introduction}

Intake system is an important part of engine, the structure parameters determined the intake flow state of engine, which affected the quality of the mixture formation and combustion quality, thus affected the overall performance of the engine. Therefore, intake system has been an important direction of the engine performance optimization. In optimizing intake system performance has made lots of research work. In theory, calculation method of one-dimensional unsteady flow model was gradually turning from characteristic line method into more efficiently finite difference method and finite volume method. In Simulation, EA 6100Q and North internal-combustion engine plant 429Q gasoline engine were used UMIST MK-14 to improve the intake and exhaust system performance, which marked the volumetric efficiency and effective power improved obviously. The paper which research object was the intake system of diesel engine was aiming at the characteristic of intake air theory, numerically simulated with AVL-BOOST. Through orthogonal experiment, it studied the effect of the intake system structure parameters on engine performance.

\section{Establishment and Analysis of Simulation Model}

The engine simulation model including cylinder, intake system, and exhaust system built with AVL-BOOST software was shown in figure 1 .

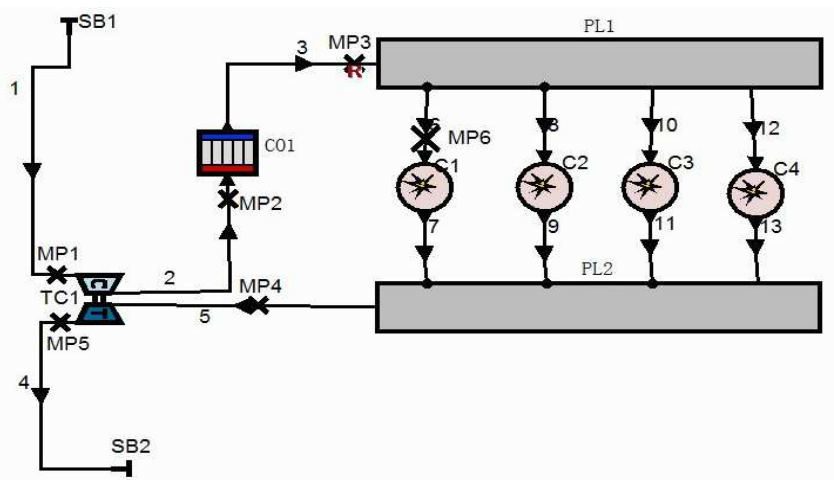

Figure 1. simulation model of AVL-BOOST

AVL-BOOT was simulated the thermal fluid with finite volume method based on the one dimensional unsteady flow. The basic control equations of the exhaust/intake system were as follows: 
Continuity equation:

$$
\frac{\partial \rho}{\partial t}+\rho \frac{\partial v}{\partial x}+v \frac{\partial \rho}{\partial x}+\frac{\rho v}{A} \frac{d A}{d x}=0
$$

Momentum equation:

$$
\frac{\partial v}{\partial t}+v \frac{\partial v}{\partial x}+\frac{\partial P}{\rho \partial x}+G=0
$$

Energy equation:

$$
\left(\frac{\partial p}{\partial t}+v \frac{\partial p}{\partial x}\right)-\frac{\kappa p}{\rho}\left(\frac{\partial \rho}{\partial t}+v \frac{\partial \rho}{\partial x}\right)-(\kappa-1) \rho(\dot{q}+v G)=0
$$

\subsection{Validation of Simulation Model}

In order to evaluate the calculation accuracy and reliability of the model shown in Figure 1, the selection of the diesel engine calibration condition was simulated calculation. The relevant experimental data is obtained in the external characteristics of the engine operating conditions. Some parameters (such as the friction coefficient) of Simulation calculation were selected by experience and reference values. The effective power and torque of the model in the calibration condition were five percent allowable error, and the fuel consumption was tended to be stable, so that the model was effective convergence. The model prediction result and was basically consistent with the actual situation of the diesel engine.

\subsection{Design and Optimization of Orthogonal Experiment}

As an effective method of mathematical statistics, orthogonal test was used not too much tests to find out the best level combination of experimental factors, and reduce blindness tests. In order to find a set of optimum parameters, Firstly, the influence degree of different parameters were obtained by the orthogonal experimental design, then established response surface model of the parameters, multi objective optimization calculation was carried out on the basis of above.

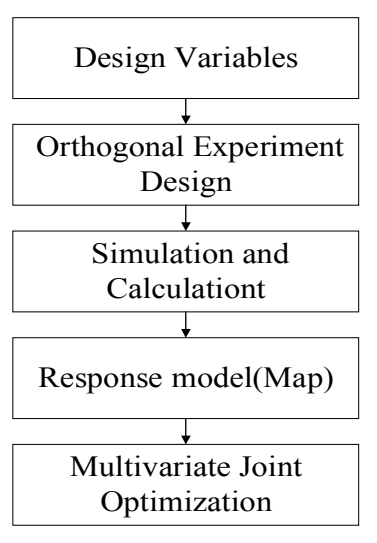

Figure 2. optimal processes

\subsection{Variables of Design and Target}

The optimization goal was to increase the engine volumetric efficiency. By optimizing the intake system structure parameters to meet constraints, the engine would reach higher volumetric efficiency.

$$
\left\{\begin{array}{l}
\max f_{\varphi}\left(L_{1}, D_{1}, L_{2}, D_{2}\right) \\
s t: \\
p \leq p_{1 \mathrm{im}} \\
\frac{d \rho}{d \phi} \leq M_{1 \mathrm{im}} \\
t \leq t_{1 \mathrm{im}} \\
f_{(H C)} \leq(H C)_{1 \mathrm{im}} \\
f_{(C O)} \leq(C O)_{1 \mathrm{im}} \\
f_{(N O x)} \leq(N O)_{1 \mathrm{im}}
\end{array}\right.
$$

$f_{\varphi}$ - Volumetric Efficiency

$L_{1}$ - Intake manifold pipe length

$L_{2}$ - Intake duck pipe length

$D_{1}$ - Intake manifold pipe diameter

$D_{2}$ - Intake duck pipe diameter

$P$-Maximum combustion pressure

$P_{\text {lim }}$-Maximum combustion pressure limit

$M_{\text {lim }}$-Growth rate of cylinder pressure limit

$\frac{d \rho}{d \varphi}$ - Growth rate of cylinder pressure

$t$-Exhaust temperature

$t_{\text {min }}$ - Exhaust temperature limit

$f_{(\text {gas })}$-Exhaust emissions

$(\text { gas })_{\lim }$-Exhaust emissions limit

\subsection{Optimization Calculation}

The test was screened in a 4 factors* 3 levels design ( $\mathrm{L}_{9}$ $\left(3^{4}\right)$ ). It only need to do nine tests, and let the computer go to work, grinding out repeated simulations with AVL-BOOST. The orthogonal experimental design method was helpful to analysis the influence of individual variables on the target, reduce the manual workload, and improve the computational efficiency.

Table 1. Experimental factor level

\begin{tabular}{ccccc}
\hline \multirow{2}{*}{ Level } & \multicolumn{4}{c}{ experimental factors } \\
\cline { 2 - 5 } & \multicolumn{2}{c}{ intake manifold } & \multicolumn{2}{c}{ intake duct } \\
\cline { 2 - 5 } & Length & Diameter & Length & Diameter \\
\hline \multirow{3}{*}{1} & $\mathrm{~A}$ & $\mathrm{~B}$ & $\mathrm{C}$ & $\mathrm{D}$ \\
2 & 100 & 40 & 100 & 30 \\
3 & 200 & 50 & 200 & 40 \\
\hline
\end{tabular}




\subsection{Optimization Analysis}

The degree of influence of each parameter on target were determine by variance analysis. The analysis of the influence of various factors on the volumetric efficiency of engine ensured the influencing order and optimal portfolio.

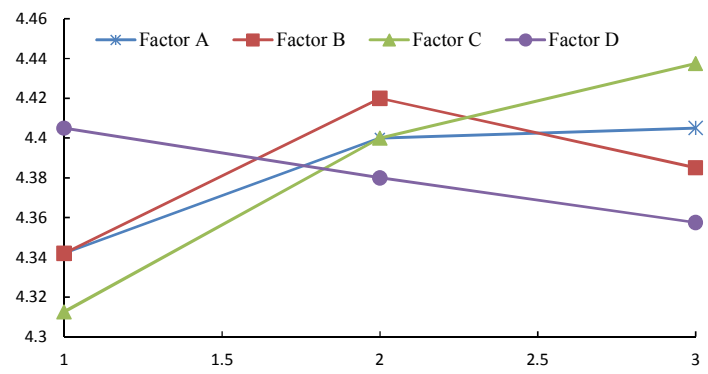

Figure 2. Order of Priority Diagram

Factor $\mathrm{C}$ affected the results highly significant. The length of the intake manifold was the biggest influence on charging efficiency. The influencing order was C, B, A, D. Response model was shown in Figure 3.

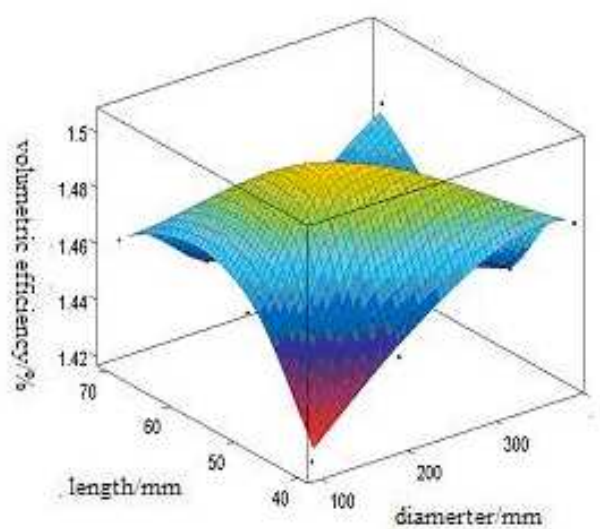

(a) Intake manifold

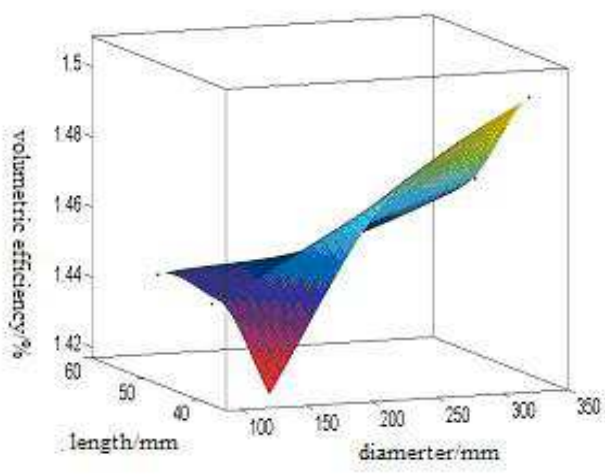

(b) Intake duct

Figure 3. Response model of intake system

In friction respect, large diameter can reduce the pressure drop, and the reduction of small diameter can increase volatility. The volumetric efficiency of the engine reached maximum value, when the intake pipe diameter was at $55 \mathrm{~mm}$. With the increase of intake duct length volumetric efficiency increased first and then decreased. Volumetric efficiency increases with the increase of intake manifold. Intake manifold diameter has little effect on charging efficiency when the intake manifold diameter was at $40 \mathrm{~mm}$. The length of the intake manifold was increased from $150 \mathrm{~mm}$ to $350 \mathrm{~mm}$, the rate of charging efficiency has been a bigger increase.

Intake system parameters were shown in table 2 before and after optimization

Table 2. Comparison of the data before and after optimization

\begin{tabular}{lcc}
\hline Data & Original & Optimized \\
\hline Length of intake manifold & 100 & 400 \\
Intake manifold Diameter & 40 & 50 \\
Length of intake duct & 100 & 200 \\
Diameter of intake duct & 30 & 30 \\
Volumetric Efficiency & 1.221 & 1.501 \\
\hline
\end{tabular}

The optimized data was actually a variety of variable compromise because of the interaction impacted of variables on the target, after optimization, the intake uniformity of each cylinder has been significantly improved, from gas uniformity is improved obviously. Intake fluctuation of Intake manifold is controlled at about $5 \%$.

The length of intake duct has a special influence on the load characteristics of the engine. Each speed has an optimal length, so the intake duct length of $100 \mathrm{~mm}, 150 \mathrm{~mm}$ and $200 \mathrm{~mm}$ were studied to analyze the relationship between intake duct pipe length and the whole machine features. The relationship was shown in Figure 4.

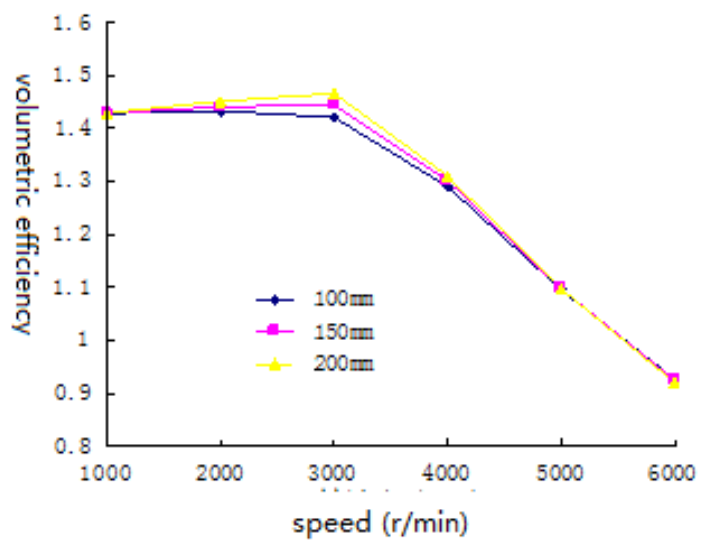

Figure 4. Impact Length of intake duck on volumetric efficiency

Along with the increase of the intake pipe length volumetric efficiency increased, when Engine speed was more than $3000 \mathrm{R} / \mathrm{min}$, the growth rate of each of the air volume reduced significantly with the increase of the length of the intake pipe.

\section{Conclusion}

(1) Working process simulation model of Engine can predict the actual engine operating conditions. 
(2) Intake system structure parameters has a different degree of impact on charging efficiency, particularly the biggest influence on it was the length of the intake manifold.

(3) Through the optimization of structural parameters of air intake pipe, a set of good structure parameters could to be found to improve charging efficiency further by the optimization of structural parameters of intake system. Air intake volatility of the intake manifold was controlled at around 5\%.

(4) The influence of different parameters on the engine performance difference was very big, so the choice of parameters was a process of compromise.

\section{Acknowledgements}

This research was supported by the modern automobile service engineering subject platform construction in Shanghai City (XKCZ1214).

The first author Ren Hongjuan was born in 1978 in Shandong province. She gained a master's degree of Power Machinery and engineering in Shandong University in 2002. Now she is an associate professor in Shanghai University of Engineering Science.

The second author Wang Huilong was born in 1988 in Shandong province. He got bachelor's degree of traffic and transportation in Liaocheng University in 2011 and now is a graduate student in Shanghai University of Engineering Science.

The third author Luo Yiping was born in 1966. He gained a master's degree of Industrial Engineering in Shanghai Jiao Tong University in 1987. Now he is a professor in Shanghai University of Engineering Science.

\section{References}

[1] Liu Yongchang, the thermodynamic process of internal combustion enginesimulation [M]. Beijing: Mechanical Industry Press, 2001

[2] Zhang Jiangcheng, Gu Hongzhong. Internal combustion engine exhaust pipe finite volume unsteady flow simulation [J]. Journal of traffic and one dimensional University. 1999(3): 339-341.

[3] Wang Shu, Liu Wentao, Li Hongmei. Experimental study on effect of intake and exhaust system on performance of flame proof diesel [J]. Coal mine machinery, 2011, 32 (5): 45-46.

[4] Li Yunqing, Zhang Jing, Li Tianlei. Numerical simulation of air flow in diesel engine [J], 2005 (6): 32-35.

[5] Yao Chunde, Fu Xiaoguang. Study on intake and exhaust system resistance performance of diesel engine on car $[\mathrm{J}]$. Small internal engine and motorcycle, 2001, 30 (5): 5-7. 